\title{
Survey Paper on Wayfarer: A Travel Recommendation System
}

\author{
Prof D.D.Gatade ${ }^{1}$, Sanket Jain ${ }^{2}$, Jay Chandarana ${ }^{3}$, Richa Mahajan ${ }^{4}$, Shweta More ${ }^{5}$ \\ Assistant Professor, Department of Computer Engineering, Sinhgad College of Engineering, Pune, India ${ }^{1}$ \\ Student, Department of Computer Engineering, Sinhgad College of Engineering, Pune, India 2,3,4,5
}

\begin{abstract}
In tourism recommendation system, a number of users and items are very large. But traditional recommendation system uses partial information for identifying similar characteristics of users. Collaborative filtering is a primary approach of any recommendation system. We are using the SentiWordNet algorithm for giving ratings to search destinations. Social networking sites act as an interactive platform for today's generation. So a lot can be extracted from social networking sites for recommendations. In the considered system, semantic positive and negative values are being calculated from SentiWordNet. Logged user can apply different filters to search destination. Captions will be extracted through social networking site and upon that text mining will be performed and trending spots will be provided to the customer through a web portal. Real-time data from Instagram is being accessed and process through spring boot. We have created APIs using RESTful web services for a web-based application.
\end{abstract}

Keywords: Representation State Transfer, Application Programming Interface.

\section{INTRODUCTION}

A Recommender system is software agents that evoke the interests and preferences of individual consumers and make recommendations accordingly. These are basically the system that recommends many things like music, videos, books, online shopping, tourism, etc. Tourism is one of a very exciting field of application of recommender systems, which is currently attracting a very high level of attention.

Their need has been largely increased because of the size of the population to which this platform cater to. They basically improved the user experience. A user, for example, would not like to go through the hassle of finding something in a very big inventory of, say Amazon and would highly appreciate if an item is being recommended to him based on some criteria like his rating of previously bought things or his most favorite category etc[5]. The huge amount of information about tourism and leisure activities available on the Web has turned the preparation of a trip into a challenging task, ripe for the website of recommender systems. Travelers are very keen on using tools that may support their decision making processes when they are planning a travel, including the choice of destination, the selection of attractions to visit, the construction of a multi-day plan, the suggestion of appropriate accommodations and restaurants, etc.

Tourism Recommender Systems usually employ a combination of diverse types of recommendation techniques: content-based, knowledge-based, collaborative filtering, demographic, etc. However, the particular characteristics of this domain lead to the continuous appearance of novel problems and the need of developing new techniques. Our recommendation system should work efficiently according to social media data. So, there exists a user interface that is suitable for travel recommendations and this interface will be a website. Once the recommendation system finds an accurate result, it will be shown on the interface to the user. In terms of hardware, recommendation system will have a database server which holds the data extracted from the social media website with another server which is responsible for creating the connection to the database and generating user specific recommendations In terms of software, our recommendation system will run on the web browsers or specific applications on personal computers, Smartphones etc. This application gathers the information from users, investigates some actions of the users, and provides the connection with the server. The server will hold the entire data in a graph database and must include all functionality to perform operations on this database, receive requests from the clients, evaluate, create and send recommendations etc. Under the operating environment of Web-based applications, MySql, internet connectivity, we are building this platform. It allows a client to search desired traveling spot according to the city name. After client enters the city name to be searched server will search all destinations in the database and displays a detailed profile of user searched destination. Logged user can also apply various filters and can recommend accordingly.

\section{RELATED WORK}

Several travel recommendation ideas based on user requirement have been proposed in the last decade. 


\section{A. Domain}

The overall idea of travel recommendation system includes data mining for the recommendation. Hence the idea of travel recommendation comes under data mining domain

\section{B. Related Study}

\section{V.K. Singh, R. Piryani, A. Uddin, P. Waila [1]}

In this paper they have devised an aspect oriented scheme that analyses the textual reviews of movies and assign it a sentiment label for each aspect [1]. The score on each aspect from multiple reviews is being aggregated and net sentiment profile of movie had generated on all parameters. They used SentiWordNet scheme to compute the document level sentiment for each movie review. The sentiment profile is also compared with document level sentiment results. They introduced two techniques SWN (AAC) and SWN (AAAVC), basically it mined a text. In SWN (AAC) only adverb and adjectives is combine and mined but in SWN (AAAVC) Adverb + Adjective and Adverb + Verb combines and mined. The SWN are proved to best then all previously used text mining algorithm. They also introduced a new sentiment named aspect-level sentiment. In this classification they keep a measure of positively and negatively expressed reviews. It also creates a aspect-vector for aspects like songs, audio, sound track or lyrics. SWN is more efficient and beneficial than alchemy API but it is only giving document level sentiment which will be recovered by aspect level sentiment. Reduce is best in terms of execution time and performance. The relative time is also reduce using map reduce technique.

We have explored different linguistic feature selection, weighing and aggregation schemes. For document-level sentiment classification, we used the SWN (AAC) and SWN (AAAVC) schemes. We obtained result of document level sentiment classification of the data collected using both these schemes and also using Alchemy API.

\section{V.Sai Supreetha, V.S.S.K.Anand [2]}

In this paper authors aims to give unified modeling language implementations on music recommender system[2]. They try to define highly abstractive UML implementations of a collaborative filtering model for music recommendation system. For developing this recommender system MVC(model view controller) architecture is followed. The artist and is represented by the play count of each user. The algorithm is applied on a Pearson correlation which help to generate similar a list of similar artists/songs to the users request. From a list generated prediction score is given which shows user likeliness towards the song. The user scenarios are used for constructing a use case diagram. Main activities for each of the services provided are described by the activity diagram. Ht e class diagram describe a structure of the system is being modeled. The sequence diagram is the one of the interaction diagrams that depicts the communication between objects. The only purpose of them is to model the music recommendation system using the behavioral and structural diagrams. They proved that UML implementation reduced the time and complexity of an algorithm.

\section{Go Hirakawa, Goshi Sato, Kenji Hisazumi, Yoshitaka Shibata [3]}

In this paper they have introduced an architecture of tourist support information system including VR contents that are aimed to promote Iwate area in Japan[3]. They proposed a system for gathering contents repository and training data to construct regional specific recommender engine on tourist support system. In tourist support system tour information server collects and manages user context, external context and social media context. The technique used by them is collaborative filtering. Recommendation engine is also implemented on server and it is connected to an android tablet client using REST API on the server. They had built a smart phone application along with this to realize navigation at the time of tour. GPS and Becon are implemented. They use training data gathering system which gathers user profile that includes implicit profile and explicit profile.

\section{Victor CODINA, and Luigi CECCARONI [4]}

Have developed a recommendation system based on semantic framework[6]. They proved that collaborative recommendation system, content-based recommendation system and few hybrid systems have some limitations which can be overcome by semantic framework. The system purposed two approaches domain-independent nature which provide a personalized services to semantic Web-applications of different domains and the recommender makes use of semantically enhanced algorithm which enhanced user modeling and content adaption. In this a domainbased method makes inferences about user's interest and a semantic similarity method is used to refine the item-user's matching algorithm. The use of FOAF-based user model help for easy integration of the recommender into a web application in any domain. In order to develop a domain-independent recommender, it is necessary to decouple the recommendation engine from the application domains. For this reason, we designed the system as a service provider following the wellknown service oriented architecture (SOA) paradigm.

In Figure 1, the abstract architectural design is represented. Using this decoupled design, each Web-application or domain has to expose a list of items to be used in the personalization process; items have to be semantically annotated using the hierarchically structured concepts of the domain ontology, which is shared with the recommender. Thus, the 
recommendation engine can work as a personalization service, providing methods to generate personalized recommendations as well as to collect user feedback while users interact with Web-applications.

In order to facilitate the reuse of user profiles as well as the authentication process we employ the widely used FOAF vocabulary as the basis of our ontologically extended user profiles, which is compatible with the OpenID authentication3. This method is a decentralized standard that allows a portable identity for users across the Web.

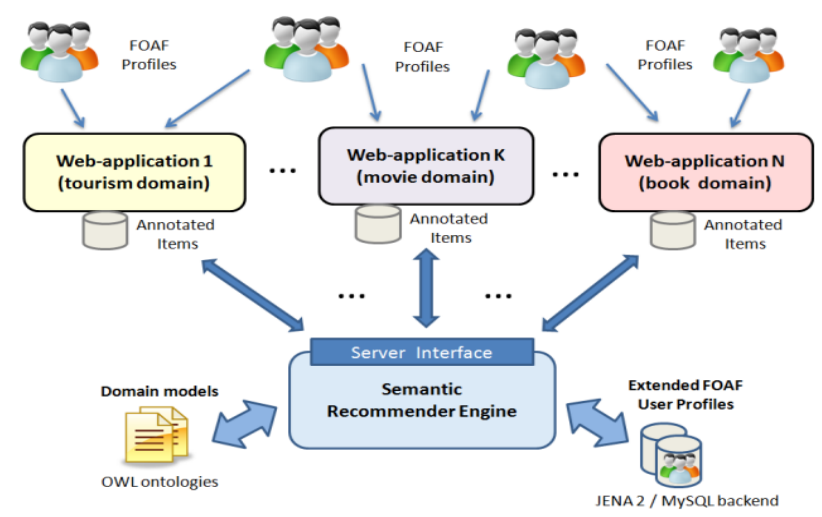

Figure 1. General architecture design

\section{Overview}

Web-based application in which a user gets the recommendation for a trending place and based on the season also user gets recommended. If a user wants to search for a specific destination they have to log in. Registered user can get the recommendation for the particular place; also can search according to various filters like budget, a particular season, destination type. Registered user can also be recommended according to searched history.

The Front-end can be accessed through REST APIs for which Springboot is used. Data is stored in MySql.

Real-time data from Instagram can be fetched and further process by Admin. Data is being updated weekly which will be stored in MySql. So media count is updated after every week which will change recommendation every week and system work on Real-time Data.

\section{CONCLUSION}

This paper demonstrates a survey on different algorithms and methods that were proposed by researchers earlier for better development in the field data mining. Real-time Instagram data is being fetched and processed in a System. In future we will be developing a new algorithm and method to create a better travelling recommendation system using data mining techniques.

\section{ACKNOWLEDGEMENT}

We would like to express our sincere gratitude towards our guide Prof. D. D. Gatade for her invaluable guidance and supervision that helped us in our research. She has always encouraged us to explore new concepts and pursue newer research problems. We credit our project contribution to her. Collectively, we would also like to thank our project committee members Prof. M. P. Wankhade and Prof. T. P. Vaidya for their time, suggestions, for graciously agreeing to be on our committee and always making themselves available. We cannot thank them enough.

\section{REFERENCES}

[1] V. K. Singh, R. Piryani, A. Uddin, P. Waila, Sentiment analysis of movie reviews, @2013 IEEE.

[2] V. Sai Supreetha, V. S. S. K. Anand, UML implementation of music recommendation system, Volume 1, Issue 8, 2014 , pp.1-7.

[3] Go Hirakawa, Goshi Sato, Kenji Hisazumi, Yoshitaka Shibata, Data gathering system for recommender system in tourism, 2015 18th International Conference on Network-Based Information Systems,2015 IEEE

[4] Victor CODINA, and Luigi CECCARONI Departament de Llenguatges i Sistemes Informàtics (LSI), Universitat Politècnica de Catalunya (UPC), Campus Nord, Edif. Omega, C. Jordi Girona, 1-3, 08034 Barcelona, Spain 